\title{
Increased Brain Functional Connectivity Correlated With Neurocognitive Performance Decline in Patients With Left Frontal Glioma
}

Masaya Ueda ( $\sim$ ueda0709@kuhp.kyoto-u.ac.jp )

Kyoto University Hospital

Kiyohide Usami

Kyoto University Graduate School of Medicine

Yukihiro Yamao

Kyoto University Graduate School of Medicine

Rie Yamawaki

Kyoto University Hospital

Chinatsu Umaba

Kyoto University Hospital

Nan Liang

Kyoto University Graduate School of Medicine

Manabu Nankaku

Kyoto University Hospital

Yohei Mineharu

Kyoto University Graduate School of Medicine

Masayuki Honda

Kyoto University Graduate School of Medicine

Takefumi Hitomi

Kyoto University Graduate School of Medicine

Ryosuke Ikeguchi

Kyoto University Graduate School of Medicine

Akio Ikeda

Kyoto University Graduate School of Medicine

Susumu Miyamoto

Kyoto University Graduate School of Medicine

\section{Shuichi Matsuda}

Kyoto University Graduate School of Medicine

Yoshiki Arakawa

Kyoto University Graduate School of Medicine 


\section{Research Article}

Keywords: glioma, electroencephalography (EEG), brain functional connectivity, neurocognitive function, left frontal lobe

Posted Date: March 3rd, 2022

DOI: https://doi.org/10.21203/rs.3.rs-1400394/v1

License: (c) (i) This work is licensed under a Creative Commons Attribution 4.0 International License.

Read Full License 


\section{Abstract}

Objectives: The association between neurocognitive decline and changing functional connectivity in glioma patients remains unclear. This study aimed to investigate the correlation between brain oscillatory activity or functional connectivity and neurocognitive function in patients with left frontal glioma using resting-state electroencephalography (EEG).

Methods: This was an observational study and conforms to all the STROBE guidelines. Participants comprised 18 consecutive patients diagnosed with newly left frontal glioma between April 2010 and March 2021 who underwent neuropsychological examination and electroencephalography. Forty seconds of artifact-free electroencephalographic data were selected, and current source density (CSD) or lagged phase synchronization (LPS) were analyzed using exact low-resolution electromagnetic tomography (eLORETA). We investigated associations of CSD and LPS with the Wechsler adult intelligence scale (WAIS), the Wechsler memory scale-revised (WMS-R), and the western aphasia battery aphasia quotient (WAB AQ) scores.

Results: Among the 18 consecutive patients (mean age, $43 \pm 13.15$ years), the mean WAIS full-scale intelligence quotient (FSIQ), WMS-R general memory (GM), and WAB AQ scores were within standard value ranges, and none had aphasia. The correlation analysis showed lower GM score was associated with higher LPS value between the right anterior prefrontal cortex and the left superior parietal lobule in the beta1 band $(13-20 \mathrm{~Hz}, \mathrm{R}=-0.802, P=0.012)$

Conclusion: Increases in functional connectivity between certain brain regions may potentially represent more common and early network changes in the brain that are associated with cognitive declines in patients with left frontal glioma.

\section{Introduction}

Gliomas are invasive brain tumors that can cause progressive neurocognitive decline. Detailed neurocognitive testing in patients with glioma reveals various cognitive deficits, including in attention, memory, language, visuospatial cognition, and executive function [1]. In particular, language-related symptoms such as speech, verbal memory, and verbal working memory in patients with left frontal glioma [2,3] may emerge through the effects of the glioma on the functional networks of the brain [2-5]. Because these kinds of neurocognitive deficit can significantly impair quality of life (QOL) and social life in patients with gliomas [6,7], understanding the mechanisms underlying neurocognitive dysfunction is clinically relevant.

Several studies using functional magnetic resonance imaging (fMRI) and magnetoencephalography (MEG) have recently shown glioma-induced changes in the resting-state functional connectivity of brain networks. Both techniques have shown that gliomas, particularly in the left hemisphere, significantly affect resting brain functional connectivity $[8,9]$. Furthermore, these changes in brain functional connectivity are correlated with changes in cognitive function among patients with glioma in the left 
hemisphere [10, 11]. MEG offers high temporal resolution, while fMRI offers high spatial resolution. However, both modalities are costly and are only applied to a small percentage of patients.

electroencephalography (EEG) is relatively inexpensive and useful for clinical follow-up. No studies appear to have investigated the relationship between functional brain connectivity and cognitive function in glioma patients using EEG.

In the present study, we assessed the local rhythmic activity and functional connectivity of the resting brain using exact low-resolution electromagnetic tomography (eLORETA) [12]. A method of nonlinear functional connectivity called "lagged phase synchronization" (LPS) [12], implemented in this eLORETA statistical package [13], is resistant to non-physiological artifacts, particularly low spatial resolution and the problem of volume conduction [14]. Furthermore, LPS can be used for filtered data, providing frequency decomposition of functional brain connectivity [12]. The physiological significance of frequency activity remains controversial, but has been suggested to be related to brain function to some extent $[15,16]$. Using these methods, an association between mini mental state examination score and theta band resting-state functional connectivity in patients with Alzheimer's disease has been reported [13].

The present study investigated the association between brain oscillatory activity or functional connectivity and neurocognitive function in patients with left frontal glioma using eLORETA. We only recruited patients with glioma located mainly in the left frontal lobe. We hypothesized that, as in previous studies, changes in brain functional connectivity between networks might correlate with changes in neurocognitive function. Our results will provide important information for determining treatment strategies, such as surgical resection and rehabilitation, in consideration of the functional outcome for the patient. This is the first study to investigate the relationship between functional connectivity and changes in cognitive function based on electroencephalographic data in patients with left frontal glioma.

\section{Methods}

\section{Ethics approval, guideline, and consent to participate}

This was an observational study and conforms to all the STROBE guidelines. The ethics committee of Kyoto University Graduate School and Faculty of Medicine (R1515) approved this study protocol. All procedures were performed in accordance with the Declaration of Helsinki. This study conforms to all Strengthening the Reporting of Observational Studies in Epidemiology guidelines. Written informed consent was obtained from patients prior to participation. Patients with a history of brain injury or drug/alcohol abuse were excluded from this study.

\section{Patients}

Participants comprised 18 consecutive patients (mean age, $43 \pm 13.15$ years) with newly diagnosed glioma in the left frontal lobe, who were treated in Kyoto University Hospital and underwent neuropsychological examination and EEG between April 2010 and March 2021. The inclusion criteria were as follows: 1) tumor located in the left frontal lobe; 2 ) tumor pathology confirmed as glioma by 
surgery; 3 ) Karnofsky performance status (KPS) score > 70 (slight disability: unable to conduct all previous activities, but able to look after own affairs without assistance); and 4) no or only minor neurological focal deficit, such as speech disturbance or paresis. Tumor pathology was confirmed at the time of surgical removal of the tumor. These characteristics are summarized in Table 1. Tumor masks of all subjects in Montreal neurological institute (MNI) space were then stacked and binarized to construct a tumor-overlapping image using the FMRIB Software Library (www.fmrib.ox.ac.uk/fsl) [17] (Fig. 1), in which each voxel was identified as part of the tumor region from at least one patient. The Edinburgh handedness inventory was used to confirm that all patients were right-handed. All patients were on antiseizure medication for control or prevention of epilepsy.

Table 1

Characteristics of patients with left frontal glioma with Karnofsky performance status (KPS)

\begin{tabular}{|llllll|}
\hline Case & Age (years) & Sex & Pathological diagnosis & WHO grade & Preoperative KPS \\
\hline 1 & 40 & F & oligodendroglioma & 2 & 100 \\
\hline 2 & 44 & F & diffuse astrocytoma & 2 & 100 \\
\hline 3 & 25 & M & oligodendroglioma & 2 & 90 \\
\hline 5 & 29 & M & oligodendroglioma & 2 & 90 \\
\hline 6 & 39 & M & diffuse astrocytoma & 2 & 100 \\
\hline 7 & 44 & M & oligodendroglioma & 2 & 80 \\
\hline 8 & 24 & M & diffuse astrocytoma & 2 & 90 \\
\hline 9 & 32 & M & oligodendroglioma & 2 & 100 \\
\hline 10 & 35 & M & diffuse astrocytoma & 2 & 100 \\
11 & 38 & M & oligodendroglioma & 2 & 100 \\
\hline 12 & 55 & M & oligodendroglioma & 2 & 100 \\
\hline 13 & 55 & F & diffuse astrocytoma & 2 & 90 \\
\hline 14 & 37 & F & diffuse astrocytoma & 2 & 100 \\
\hline 15 & 54 & F & anaplastic oligodendroglioma & 3 & 100 \\
\hline 16 & 53 & M & anaplastic astrocytoma & 3 & 90 \\
\hline 17 & 72 & F & anaplastic oligodendroglioma & 3 & 100 \\
\hline 18 & 63 & F & Glioblastoma & 4 & 90 \\
\hline Sex: F $=$ female; M = male. WMS-R scores: WHO = world health organization. KPS & Karnofsky \\
performance status. & & & & \\
\hline
\end{tabular}




\section{Evaluation of neurocognitive function}

Thirteen patients finished evaluation of neurocognitive function before starting treatment for glioma, the Wechsler adult intelligence scale-revised (WAIS-R, $n=1)$, WAIS-III $(n=9)$, or WAIS-IV $(n=6)$, Wechsler memory scale-revised (WMS-R) and Western Aphasia Battery (WAB). In addition, some patients and their families reported loss of attention, decreases in memory, performance difficulty, or minor changes of characteristics. Only one subject (Case 4) could not finish the WAIS due to a lack of time available for testing.

\section{EEG recordings and data acquisition}

All patients underwent recording of 30 min of EEG for clinical purposes. This study analyzed $40 \mathrm{~s}$ of resting-state data from that EEG data from the 18 patients with left frontal glioma. We only used data obtained during the resting state with eyes closed and in an arousal state. A certified clinical neurophysiologist (K.U.) checked the findings and confirmed that interictal epileptic discharges or continuous focal slow waves were excluded from the analysis windows.

EEG was recorded with a digital 19-channel scalp EEG device, using the International 10-20 system (i.e., Fp1, Fp2, F7, F3, Fz, F4, F8, T7, C3, Cz, C4, T8, P7, P3, Pz, P4, P8, 01, 02). EEG data were acquired with a linked ear reference, sampled at $500 \mathrm{~Hz}$, and filtered off-line between $0.53 \mathrm{~Hz}$ and $60 \mathrm{~Hz}$. Electrode impedance was kept below $5 \mathrm{k} \Omega$. EEG recording included eyes open and closed states with vigilance control. For all patients, we selected $40 \mathrm{~s}$ of artifact-free EEG data, fragmented off-line into 2-s segments. EEG artifacts were manually excluded, and EEG findings were clinically analyzed by certified electroencephalographers. The EEG data used for the following analyses excluded findings suggestive of focal abnormality (focal slow waves) or interictal epileptic activities (spike or sharp waves). Segments including blink artifact, muscle artifact, electrocardiographic artifact, or signs of drowsiness were rejected, and only reliable, awake EEG data were selected to ensure that we could adequately estimate brain function during resting-state. EEG data were analyzed using the LORETA-KEY software package (product by The KEY Institute for Brain-Mind Research).

\section{EEG source localization}

We investigated the cortical distribution of the current source density (CSD) using eLORETA, which calculates CSD as neuronal electrical activity without assuming a specific number of active sources. The head model in eLORETA and the electrode coordinates is based on the MNI average MRI brain map (MNI152) [18]. The solution space was limited to the cortical gray matter, including 6239 voxels with a spatial resolution of $5 \mathrm{~mm}^{3}$. Previous studies have validated eLORETA tomography using fMRI $[19,20]$, structural MRI [21], positron emission tomography (PET) [22] and intracranial EEG [23]. In this study, intracerebral electrical sources that yielded scalp-recorded potentials in each frequency band were estimated using CSD values. Selected artifact-free EEG fragments were analyzed to calculate the 
eLORETA cortical CSD from $0.53 \mathrm{~Hz}$ to $30 \mathrm{~Hz}$. CSD of the eLORETA cortical functioning image was calculated for 6 frequency bands: delta, 2-4 Hz; theta, 4-8 Hz; alpha1, 8-10 Hz; alpha2, 10-13 Hz; beta1, 13-20 Hz; and beta2, 20-30 Hz.

\section{Functional connectivity analysis}

To analyze functional connectivity, we adopted a voxel-wise approach to determine cortical regions of interest (ROI). With eLORETA, MNI coordinates of the cortical voxels underlying the electrode sites were defined to create the ROIs. Based on previous research on functional connectivity [24], 21 ROIs were selected. Three additional ROls comprising two auditory fields and visual fields were included, as these have recently attracted attention in studies on brain functional networks $[25,26]$. As a result, we defined a total of 24 cortical ROls (Table 2). 
Table 2

The 24 cerebral regions of interest (ROIs) determined by eLORETA

\begin{tabular}{|c|c|c|c|c|}
\hline \multirow[t]{2}{*}{ Anatomical region } & \multirow[t]{2}{*}{ Brodmann area } & \multicolumn{3}{|c|}{ ROI centroid MNI coordinates } \\
\hline & & $x$ & $y$ & $z$ \\
\hline Left middle temporal area (IMT) & 37 & -50 & -70 & -5 \\
\hline Right middle temporal area (rMT) & 37 & 45 & 70 & -5 \\
\hline Left frontal eye fields (IFEF) & 6 & -25 & -10 & 50 \\
\hline Right frontal eye fields (rFEF) & 6 & 25 & -10 & 50 \\
\hline Left superior parietal lobule (ISPL) & 7 & -25 & -50 & 55 \\
\hline Right superior parietal lobule (rSPL) & 7 & 25 & -50 & 55 \\
\hline Left anterior prefrontal cortex (laPFC) & 10 & -35 & 55 & 5 \\
\hline Right anterior prefrontal cortex (raPFC) & 10 & 35 & 55 & 5 \\
\hline Left dorsolateral prefrontal cortex (IdIPFC) & 9 & -50 & 15 & 40 \\
\hline Right dorsolateral prefrontal cortex (rdlPFC) & 9 & 50 & 15 & 40 \\
\hline Anterior cingulate cortex (aCC) & 32 & 5 & 30 & 25 \\
\hline Left anterior inferior parietal lobule (laIPL) & 40 & -50 & -50 & 45 \\
\hline Right anterior inferior parietal lobule (ralPL) & 40 & 50 & -50 & 45 \\
\hline Left anterior insula (laINS) & 47 & -30 & 20 & -5 \\
\hline Right anterior insula (ralNS) & 47 & 30 & 20 & -5 \\
\hline Left hippocampal formation (IHF) & 28 & -20 & -20 & -20 \\
\hline Right hippocampal formation (rHF) & 28 & 20 & -20 & -20 \\
\hline Ventromedial prefrontal cortex (vmPFC) & 10 & -5 & 50 & -5 \\
\hline Posterior cingulate cortex (pcC) & 23 & 0 & -55 & 15 \\
\hline Left posterior inferior parietal lobule (IpIPL) & 39 & -50 & -70 & 30 \\
\hline Right posterior inferior parietal lobule (rpIPL) & 39 & 50 & -70 & 30 \\
\hline Visual fields (Vis) & 18 & 0 & -90 & -5 \\
\hline Left auditory fields (IAud) & 41 & -55 & -25 & 10 \\
\hline Right auditory fields (rAud) & 41 & 55 & -25 & 10 \\
\hline
\end{tabular}


Although detailed information on the eLORETA connectivity algorithm has recently been published elsewhere [12, 27], we briefly summarize this method here. To analyze functional connectivity between all pairs of ROls, we used LPS. This is a method for evaluating similarities between signals in the frequency domain, based on normalized Fourier transforms. LPS is thus associated with nonlinear functional connectivity. This lagged connectivity measure is considered to be accurately corrected as it represents the connectivity of two signals after excluding the instantaneous zero-lag component (i.e., several artifact elements). Such correction is necessary because scalp EEG signals or estimated intracranial signals (EEG tomography) often include non-physiological components or physical artifacts, such as volume conduction that usually affects other connectivity indices. LPS is thus considered to include only physiological connectivity information.

\section{Statistical analyses}

We assessed the associations between EEG parameters (i.e., CSD and LPS values) and three neurocognitive function scores, i.e, WAIS full-scale intelligence quotient (FSIQ) score, WMS-R generalized memory (GM) scores, and WAB aphasia quotient (AQ) by using the eLORETA software. For the statistical analysis, eLORETA applies a statistical nonparametric mapping method (SnPM), which have demonstrated to be effective in controlling the Type I error in neuroimaging studies [28]. eLORETA used 5000 data randomizations with correction for multiple comparisons across all voxels and all frequencies. The application of SnPM for LORETA images has been validated in several studies [29, 30].

The critical probability threshold for $P$ values was set at $P=.05$, and determined by a SnPM with correction for multiple comparisons across all frequencies. In the further step, the results of correlation analysis for the three neurocognitive scores in multiple tests were corrected using the Bonferroni method. Specifically, the Bonferroni method was used to determine the adjusted significance level $(P<0.05 / 3=$ 0.017), and a judgment was made at $P<0.017$ for the probability value of the correlation test results for each analysis. In addition, the correlations between the sub-items of the neurocognitive function and the EEG parameters were also analyzed when significant correlations were detected between the EEG parameters and neurocognitive function.

\section{Results}

\section{Neurocognitive performance}

Results of evaluation of neurocognitive function are listed in Table 3. Mean ( \pm standard deviation) scores were as follows: FSIQ, $101.24 \pm 14.42$; GM, 103.83 \pm 16.13 ; and WAB AQ, $98.8 \pm 1.32$. Based on WAIS FSIQ scores, 3 patients were considered to have experienced global intelligence decline (No.13, 80; No. 14, 72; No.18, 77). Based on WMS-R GM scores, 4 patients were considered to have experienced memory declines (No. 4, 73; No. 7, 84; No. 14, 78; No. 18, 82). However, no patients showed definitive aphasia as based on WAB AQ scores. 
Table 3

Results of evaluation of neurocognitive function of all patients

\begin{tabular}{|lllllllllllll|}
\hline Case & WAIS & & \multicolumn{7}{c}{ WMS-R } & & & WAB \\
\hline & FSIQ & VCI & POI & WMI & PSI & VeM & ViM & GM & A/C & DR & AQ \\
\hline 1 & 117 & 120 & 121 & 103 & 84 & 110 & 120 & 114 & 126 & 124 & 99 \\
\hline 2 & 96 & 104 & 89 & 94 & 102 & 115 & 119 & 118 & 102 & 112 & 100 \\
\hline 3 & 115 & 106 & 122 & 119 & 102 & 109 & 121 & 114 & 134 & 113 & 98.1 \\
\hline 4 & NA & NA & NA & NA & NA & 66 & 104 & 73 & 95 & 69 & 98.4 \\
\hline 5 & 107 & 105 & 108 & 102 & 100 & 102 & 120 & 107 & 103 & 110 & 100 \\
\hline 6 & 107 & 100 & 125 & 107 & 94 & 87 & 115 & 94 & 132 & 105 & 99.6 \\
\hline 7 & 87 & NA & NA & NA & NA & 74 & 118 & 84 & 91 & 94 & 100.0 \\
\hline 8 & 100 & 92 & 114 & 103 & 89 & 109 & 105 & 99 & 94 & 114 & 99.2 \\
\hline 9 & 96 & 107 & 93 & 81 & 75 & 115 & 104 & 113 & 89 & 99 & 100.0 \\
\hline 10 & 110 & 116 & 103 & 98 & 97 & 116 & 104 & 114 & 92 & 97 & 99.6 \\
\hline 11 & 105 & 111 & 114 & 85 & 84 & 97 & 101 & 98 & 104 & 98 & 97.7 \\
\hline 12 & 72 & 85 & 85 & 67 & 68 & 68 & 108 & 78 & 76 & 85 & 98.5 \\
\hline 13 & 116 & 110 & 124 & 114 & 105 & 104 & 113 & 108 & 114 & 114 & 100 \\
\hline 14 & 114 & 107 & 128 & 109 & 102 & 110 & 122 & 115 & 120 & 120 & 98.9 \\
\hline 15 & 80 & 77 & 97 & 76 & 82 & 106 & 108 & 107 & 92 & 90 & 96.8 \\
\hline 16 & 109 & 115 & 124 & 100 & 79 & 132 & 119 & 132 & 102 & 127 & 100 \\
\hline 17 & 113 & 110 & 103 & 117 & 118 & 123 & 105 & 119 & 123 & 118 & 96.8 \\
\hline 18 & 77 & 99 & 87 & 58 & 78 & 82 & 90 & 82 & 94 & 82 & 95.7 \\
\hline WAIS scores: FSIQ $=$ full-scale intelligence quotient; VCI = verbal comprehension index; POI = \\
perceptual organization index; WMI = working memory index; PSI = processing speed index. WMS-R \\
\hline Acores: Verbal memory = verbal memory; Visual memory = visual memory; GM = generalized memory;
\end{tabular}

\section{Association between CSD and evaluation of neurocognitive function scores}

The highest CSD values were found in the alpha1 band in the left cuneus occipital lobe (CSD value, 366), followed by the delta band at the medial frontal gyrus (CSD value, 336). We investigated the association between CSD values and WAIS FSIQ scores, WMS-R GM scores and WAB AQ scores in patients with left 
frontal glioma. All 6239 cortical voxels were explored in CSD analysis. The CSD analyzed showed no significant correlation between CDS and all cognitive examination scores.

\section{Association between LPS and neurocognitive function scores}

Results of analyses for association between LPS values and WAIS FSIQ scores, WMS-R GM scores and WAB AQ scores by eLORETA showed a significant correlation with GM in the beta1 band (Fig. 2). GM scores showed a strong negative correlation with LPS value between right anterior prefrontal cortex (raPFC) and left superior parietal lobule (ISPL) in the beta1 band ( $\mathrm{R}=-0.802, P=0.012)$; the lower the $\mathrm{GM}$ scores, the higher the LPS values between these regions. In addition, the correlations between the subitems of WMS-GM (verbal memory, $101.39 \pm 18.79$; visual memory, $110.89 \pm 8.94$; attention/concentration, $104.61 \pm 16.51$; delayed memory, $103.94 \pm 15.79$ ) scores and LPS values were analyze. Interestingly, post hoc analysis indicated that verbal memory score has negative correlation with LPS between raPFC and ISPL in the beta1 band $(\mathrm{R}=-0.837, P=0.004)(\mathrm{Fig} .2)$.

\section{Discussion}

This study investigated the associations between resting-state EEG parameters (i.e., CSD and LPS values) and neurocognitive functions among patients with glioma in the left frontal lobe. 3 patients showed a FSIQ decline, and 4 patients had a GM decline. Nevertheless, mean WAIS FSIQ and WMS-R GM were within the standard (FSIQ ranged from 86.62 to 115.66 and GM 87.7 to 119.96) [31, 32], mean WAB AQ scores was more than cutoff point (93.8) [33]. GM and verbal memory scores showed a strong negative correlation with LPS value between raPFC and ISPL in the beta1 band $(13-20 \mathrm{~Hz})$.

The patients with left frontal glioma often had some language function decline [1]. Notably, no clear decrease in language function was seen in any subjects with included language-related brain regions in this study. Resting-state fMRI studies have shown that compensation such as increased functional connectivity in the cerebellum and language-related regions of the right hemisphere occurs in patients with left-brain glioma [34]. Our results for neurocognitive performance indicated that other neurocognitive functions may be impaired even in the absence of language impairment.

Analysis for associations between CSD and neurocognitive function scores showed no significant correlation. The characteristics and distribution of electroencephalographic changes produced by a tumor primarily depend on the size of the lesion, its rate of growth, its distance from the cortical surface, and the tumor type [35]. In particular, when brain tumors are located superficially, focal slow waves can be observed on scalp EEG [35]. In this study, the tumor sizes of left frontal lobe glioma were heterogeneous and distance from the brain surface and location of the lesion varied from patient to patient. Nevertheless, most of the electroencephalographic data used in the analysis did not include slow activities that indicate localized abnormalities. Thus, CSD for our subjects included fewer individual differences, which may be a reason why no correlations with cognitive function were identified. 
Remarkably, GM and verbal memory score correlated inversely with functional connectivity between the raPFC and ISPL. The GM score is the sum of the verbal memory and visual memory scores. The post hoc analysis showed that this change in functional connectivity was more related to verbal memory than visual memory. In glioma patients, changes in functional connectivity are reportedly associated with changes in cognitive function according to recent studies [11,36]. Our results differed in that the correlation was negative and showed beta1 band connectivity. Brain functional connectivity is pathologically enhanced by aging and neurological diseases and is associated with worsening symptoms and cognitive decline [37-39]. Lockhart showed that cognitive performance in healthy older adults correlated inversely with increased functional connectivity in the frontal lobes, and argued that this result was a "toxic consequence" rather than a compensatory connection [38]. In fact, several studies have suggested that increased connectivity reduces the efficiency of information transfer and does not ultimately aid in cognitive function $[40,41]$. The negative correlations observed in this study may also reflect such pathological changes.

Another significant aspect of the results is the functional connectivity associated with verbal memory was extending to the contralateral hemisphere. Resting-state fMRI studies in patients with glioma have indicated that inter-network connections were stronger than in healthy controls and were often observed in unexpected pairs and regions [10]. Previous studies in function of front-parietal connectivity have reported, connectivity between medial aPFC and the right central precuneus and intraparietal sulcus/inferior parietal lobule associated metacognitive ability for memory retrieval [42]. It also emphasizes the relationship between parietal-frontal connectivity and impaired updating and manipulation of contextual information and delusions, mainly in schizophrenic patients [43, 44]. Hence, it could be hypothesized that glioma affect wide extensive inter-network connectivity which associate with neurocognitive function.

Several previous studies have reported that functional connectivity in the resting alpha band is linearly associated with improvable misorients in language and cognitive functions in stroke patients and glioma patients $[45,46]$. Differences in the characteristics of subjects may explain the gap between our results and the findings of those previous studies. Our subjects displayed less cognitive decline (present in only 4 of 18 subjects) compared to previous studies. In addition, tumor location has been reported to significantly affect cognitive function, 1 and our study included only subjects in whom the left frontal lobe was the main location of the lesion. We may therefore have found more common early network changes in the brain, which are associated with cognitive declines in glioma patients. These findings, such as those from routine scalp EEG data, are clinically useful, and suggest that assessment of brain functional connectivity between networks is an important issue in the search for clinical markers of early-stage glioma.

We should specify some limitations to the present research. First, although the locations of the lesions were relatively similar, the world health organization (WHO) grade of tumor (13 patients with low-grade tumor, 5 patients with high-grade tumor), tumor size, and degree of progression may have differed. In patients with glioblastoma, slow waves are more likely to be seen in lesions closer to the brain surface,35 
which may have affected individual differences in EEG data. Second, since the number of electrodes was small (19 channels), discussion of localization requires careful interpretation. We used eLORETA to analyze brain oscillatory activity and functional connectivity to resolve problems of spatial resolution. However, using eLORETA has methodological limitations in exploring brain function. For example, despite the proven utility and reproducibility, eLORETA results rely on an inferred model and may not accurately represent the origin of brain activity.

\section{Conclusion}

Evaluation of neurocognitive function in patients with left frontal glioma showed other cognitive deficits even in the absence of marked aphasia. In addition, analysis of neurocognitive function and functional brain connectivity showed a negative correlation between general memory and verbal memory and functional connectivity in the beta1 band of the right anterior prefrontal cortex and the left superior parietal lobule. We may have found more common and early network changes in the brain that are associated with cognitive decline in patients with left frontal glioma. Findings such as those from routine scalp EEG are clinically useful, and the present results suggest that assessment of brain functional connectivity between networks is an important issue in the search for clinical markers of early-stage glioma.

\section{Abbreviations}

$\mathrm{AQ}=$ aphasia quotient

$\mathrm{CSD}=$ current source density

$\mathrm{EEG}=$ electroencephalography

eLORETA = exact low-resolution electromagnetic tomography

$\mathrm{fMRI}=$ functional magnetic resonance imaging

LPS = lagged phase synchronization

ISPL = left superior parietal lobule

$\mathrm{FSIQ}=$ full-scale intelligence quotient

$\mathrm{GM}=$ general memory

KPS $=$ Karnofsky performance status

MEG = magnetoencephalography

$\mathrm{MNI}=$ Montreal neurological institute 
$\mathrm{PET}=$ positron emission tomography

$\mathrm{QOL}$ = quality of life

raPFC = right anterior prefrontal cortex

$\mathrm{ROI}=$ region of Interest

$\mathrm{WAB}=$ western aphasia battery

WAIS $=$ Wechsler adult intelligence scale

$\mathrm{WHO}=$ world health organization

WMS-R $=$ Wechsler memory scale-revised

\section{Declarations}

\section{Acknowledgments}

We wish to thank the staff of Kyoto University Hospital for their assistance during awake craniotomy. We are also grateful to Yasuo Naito and Ryouhei Ishii from Osaka Prefecture University and Masahiro Hata from Osaka University for their assistance with the statistical analyses.

\section{Competing Interests}

The authors report no conflicts of interest concerning the materials or methods used in this study or the findings specified in this paper.

\section{Author contributions}

Conception and design: Ueda, Usami, Arakawa. Acquisition of data: Ueda, Usami, Yamao, Yamawaki, Umaba, Liang, Nankaku, Honda, Hitomi. Analysis and interpretation of data: Ueda, Usami, Yamao, Yamawaki. Drafting the article: Ueda, Usami, Yamawaki. Critically revising the article: Mineharu, Ikeda, Ikeguchi, Matsuda, Miyamoto, Arakawa. Reviewed submitted version of manuscript: all authors. Statistical analysis: Ueda, Usami. Administrative/technical/material support: Usami, Yamao, Honda, Hitomi. Study supervision: Arakawa.

\section{Study funding}

This work was supported by grants-in-aid from the Ministry of Education, Culture, Sports, Science, and Technology, Japan (16K10754, 20K16492).

\section{Data availability}


Anonymized data used in the present study are available upon reasonable request to the corresponding author.

\section{References}

1. Satoer D, Visch-Brink E, Dirven C, Vincent A. Glioma surgery in eloquent areas: can we preserve cognition?. Acta Neurochir (Wien). 2016;158(1):35-50. doi:10.1007/s00701-015-2601-7

2. Bello L, Gallucci M, Fava M, et al. Intraoperative subcortical language tract mapping guides surgical removal of gliomas involving speech areas. Neurosurgery. 2007;60(1):67-82. doi:10.1227/01.NEU.0000249206.58601.DE

3. Papagno C, Casarotti A, Comi A, Gallucci M, Riva M, Bello L. Measuring clinical outcomes in neurooncology. A battery to evaluate low-grade gliomas (LGG). J Neurooncol. 2012;108(2):269-275. doi:10.1007/s11060-012-0824-5

4. King TZ, Na S, Mao H. Neural Underpinnings of Working Memory in Adult Survivors of Childhood Brain Tumors. J Int Neuropsychol Soc. 2015;21(7):494-505. doi:10.1017/S135561771500051X

5. Robinson KE, Pearson MM, Cannistraci CJ, et al. Functional neuroimaging of working memory in survivors of childhood brain tumors and healthy children: Associations with coping and psychosocial outcomes. Child Neuropsychol. 2015;21(6):779-802. doi:10.1080/09297049.2014.924492

6. Klein M, Taphoorn MJ, Heimans JJ, et al. Neurobehavioral status and health-related quality of life in newly diagnosed high-grade glioma patients [published correction appears in J Clin Oncol. $2003 \mathrm{Jul}$ 1;21(13):2628]. J Clin Oncol. 2001;19(20):4037-4047. doi:10.1200/JC0.2001.19.20.4037

7. Taphoorn MJ, Sizoo EM, Bottomley A. Review on quality of life issues in patients with primary brain tumors. Oncologist. 2010;15(6):618-626. doi:10.1634/theoncologist.2009-0291

8. Ghumman S, Fortin D, Noel-Lamy M, Cunnane SC, Whittingstall K. Exploratory study of the effect of brain tumors on the default mode network. J Neurooncol. 2016;128(3):437-444. doi:10.1007/s11060016-2129-6 9.

9. Harris RJ, Bookheimer SY, Cloughesy TF, et al. Altered functional connectivity of the default mode network in diffuse gliomas measured with pseudo-resting state fMRI. J Neurooncol. 2014;116(2):373-379. doi:10.1007/s11060-013-1304-2

10. Kinno R, Ohta S, Muragaki Y, Maruyama T, Sakai KL. Differential reorganization of three syntaxrelated networks induced by a left frontal glioma. Brain. 2014;137(Pt 4):1193-1212. doi:10.1093/brain/awu013

11. Maesawa S, Bagarinao E, Fujii M, et al. Evaluation of resting state networks in patients with gliomas: connectivity changes in the unaffected side and its relation to cognitive function. PLOS One. 2015;10(2):e0118072. Published 2015 Feb 6. doi:10.1371/journal.pone.0118072

12. Pascual-Marqui RD, Lehmann $D$, Koukkou $M$, et al. Assessing interactions in the brain with exact lowresolution electromagnetic tomography. Philos Trans A Math Phys Eng Sci. 2011;369(1952):3768- 
3784. doi:10.1098/rsta.2011.0081

13. Hata M, Kazui H, Tanaka T, et al. Functional connectivity assessed by resting state EEG correlates with cognitive decline of Alzheimer's disease - An eLORETA study. Clin Neurophysiol. 2016;127(2):1269-1278. doi:10.1016/j.clinph.2015.10.030

14. Stam CJ, Nolte G, Daffertshofer A. Phase lag index: assessment of functional connectivity from multi channel EEG and MEG with diminished bias from common sources. Hum Brain Mapp. 2007;28(11):1178-1193. doi:10.1002/hbm.20346

15. Uhlhaas PJ, Haenschel C, Nikolić D, Singer W. The role of oscillations and synchrony in cortical networks and their putative relevance for the pathophysiology of schizophrenia. Schizophr Bull. 2008;34(5):927-943. doi:10.1093/schbul/sbn062

16. Uhlhaas PJ, Singer W. Abnormal neural oscillations and synchrony in schizophrenia. Nat Rev Neurosci. 2010;11(2):100-113. doi:10.1038/nrn2774

17. Smith SM, Jenkinson M, Woolrich MW, et al. Advances in functional and structural MR image analysis and implementation as FSL. Neuroimage. 2004;23 Suppl 1:S208-S219. doi:10.1016/j.neuroimage.2004.07.051

18. Mazziotta J, Toga A, Evans A, et al. A probabilistic atlas and reference system for the human brain: International Consortium for Brain Mapping (ICBM). Philos Trans R Soc Lond B Biol Sci. 2001;356(1412):1293-1322. doi:10.1098/rstb.2001.0915

19. Mulert C, Jäger L, Schmitt R, et al. Integration of fMRI and simultaneous EEG: towards a comprehensive understanding of localization and time-course of brain activity in target detection. Neuroimage. 2004;22(1):83-94. doi:10.1016/j.neuroimage.2003.10.051

20. Vitacco D, Brandeis D, Pascual-Marqui R, Martin E. Correspondence of event-related potential tomography and functional magnetic resonance imaging during language processing. Hum Brain Mapp. 2002;17(1):4-12. doi:10.1002/hbm.10038

21. Worrell GA, Lagerlund TD, Sharbrough FW, et al. Localization of the epileptic focus by low-resolution electromagnetic tomography in patients with a lesion demonstrated by MRI. Brain Topogr. 2000;12(4):273-282. doi:10.1023/a:1023407521772

22. Dierks T, Jelic V, Pascual-Marqui RD, et al. Spatial pattern of cerebral glucose metabolism (PET) correlates with localization of intracerebral EEG-generators in Alzheimer's disease. Clin Neurophysiol. 2000;111(10):1817-1824. doi:10.1016/s1388-2457(00)00427-2

23. Zumsteg D, Friedman A, Wieser HG, Wennberg RA. Propagation of interictal discharges in temporal lobe epilepsy: correlation of spatiotemporal mapping with intracranial foramen ovale electrode recordings. Clin Neurophysiol. 2006;117(12):2615-2626. doi:10.1016/j.clinph.2006.07.319

24. Vincent JL, Kahn I, Snyder AZ, Raichle ME, Buckner RL. Evidence for a frontoparietal control system revealed by intrinsic functional connectivity [published correction appears in J Neurophysiol. 2011 Mar;105(3):1427]. J Neurophysiol. 2008;100(6):3328-3342. doi:10.1152/jn.90355.2008

25. Pascual B, Masdeu JC, Hollenbeck M, et al. Large-scale brain networks of the human left temporal pole: a functional connectivity MRI study. Cereb Cortex. 2015;25(3):680-702. 
doi:10.1093/cercor/bht260

26. Li R, Wang S, Zhu L, et al. Aberrant functional connectivity of resting state networks in transient ischemic attack. PLoS One. 2013;8(8):e71009. Published 2013 Aug 12.

doi:10.1371/journal.pone.0071009

27. Canuet L, Ishii R, Pascual-Marqui RD, et al. Resting-state EEG source localization and functional connectivity in schizophrenia-like psychosis of epilepsy. PLoS One. 2011;6(11):e27863.

doi:10.1371/journal.pone.0027863

28. Nichols TE, Holmes AP. Nonparametric permutation tests for functional neuroimaging: a primer with examples. Hum Brain Mapp. 2002;15(1):1-25. doi:10.1002/hbm.1058

29. Pascual-Marqui RD, Lehmann D, Koenig T, et al. Low resolution brain electromagnetic tomography (LORETA) functional imaging in acute, neuroleptic-naive, first-episode, productive schizophrenia. Psychiatry Res. 1999;90(3):169-179. doi:10.1016/s0925-4927(99)00013-x

30. Anderer P, Pascual-Marqui RD, Semlitsch HV, Saletu B. Electrical sources of P300 event-related brain potentials revealed by low resolution electromagnetic tomography. 1. Effects of normal aging. Neuropsychobiology. 1998;37(1):20-27. doi:10.1159/000026472

31. Wechsler D. Administration and Scoring Manual for the Wechsler Adult Intelligence Scale Technical manual for the Wechsler Adult Intelligence Scale. Harcourt Assessment, Inc. San Antonio, Texas; 1997.

32. D. Wechsler, WMS-R: Wechsler Memory Scale-Revised manual. The Psychological Corporation. San Antonio, Texas; 1987.

33. Kertesz A. Western Aphasia Battery test manual. NY: Grune \& Stratton. New York; 1982.

34. Yuan B, Zhang N, Yan J, Cheng J, Lu J, Wu J. Resting-state functional connectivity predicts individual language impairment of patients with left hemispheric gliomas involving language network. Neuroimage Clin. 2019;24:102023. doi:10.1016/j.nicl.2019.102023

35. Fischer-Williams M, Dike GL. Brain tumors and other space-occupying lesions. Niedermeyer E, DaSilva FL, eds. Electroencephalography: Basic Principles, Clinical Applications, and Related Fields. 3rd ed. Williams \& Wilkins; 1993. 305-432.

36. Lang S, Gaxiola-Valdez I, Opoku-Darko M, et al. Functional Connectivity in Frontoparietal Network: Indicator of Preoperative Cognitive Function and Cognitive Outcome Following Surgery in Patients with Glioma. World Neurosurg. 2017;105:913-922.e2. doi:10.1016/j.wneu.2017.05.149

37. Chand GB, Wu J, Hajjar I, Qiu D. Interactions of the Salience Network and Its Subsystems with the Default-Mode and the Central-Executive Networks in Normal Aging and Mild Cognitive Impairment. Brain Connect. 2017;7(7):401-412. doi:10.1089/brain.2017.0509

38. Chen H, Li Y, Liu Q, et al. Abnormal Interactions of the Salience Network, Central Executive Network, and Default-Mode Network in Patients With Different Cognitive Impairment Loads Caused by Leukoaraiosis. Front Neural Circuits. 2019;13:42. Published 2019 Jun 18.

doi:10.3389/fncir.2019.00042

Page $17 / 20$ 
39. Lockhart SN, Luck SJ, Geng J, et al. White matter hyperintensities among older adults are associated with futile increase in frontal activation and functional connectivity during spatial search. PLoS One. 2015;10(3):e0122445. Published 2015 Mar 20. doi:10.1371/journal.pone.0122445

40. Medaglia JD, Chiou KS, Slocomb J, et al. The less BOLD, the wiser: support for the latent resource hypothesis after traumatic brain injury. Hum Brain Mapp. 2012;33(4):979-993. doi:10.1002/hbm.21264

41. Hillary FG, Grafman JH. Injured Brains and Adaptive Networks: The Benefits and Costs of Hyperconnectivity. Trends Cogn Sci. 2017;21(5):385-401. doi:10.1016/j.tics.2017.03.003

42. Baird B, Smallwood J, Gorgolewski KJ, Margulies DS. Medial and lateral networks in anterior prefrontal cortex support metacognitive ability for memory and perception. $J$ Neurosci. 2013;33(42):16657-16665. doi:10.1523/JNEUROSCI.0786-13.2013.

43. Kaplan CM, Saha D, Molina JL, et al. Estimating changing contexts in schizophrenia. Brain. 2016;139(Pt 7):2082-2095. doi:10.1093/brain/aww095

44. Baker SC, Konova AB, Daw ND, Horga G. A distinct inferential mechanism for delusions in schizophrenia. Brain. 2019;142(6):1797-1812. doi:10.1093/brain/awz051

45. Bosma I, Stam CJ, Douw L, et al. The influence of low-grade glioma on resting state oscillatory brain activity: a magnetoencephalography study. J Neurooncol. 2008;88(1):77-85. doi:10.1007/s11060008-9535-3

46. Sadaghiani S, Kleinschmidt A. Brain Networks and a-Oscillations: Structural and Functional Foundations of Cognitive Control. Trends Cogn Sci. 2016;20(11):805-817. doi:10.1016/j.tics.2016.09.004

\section{Figures}




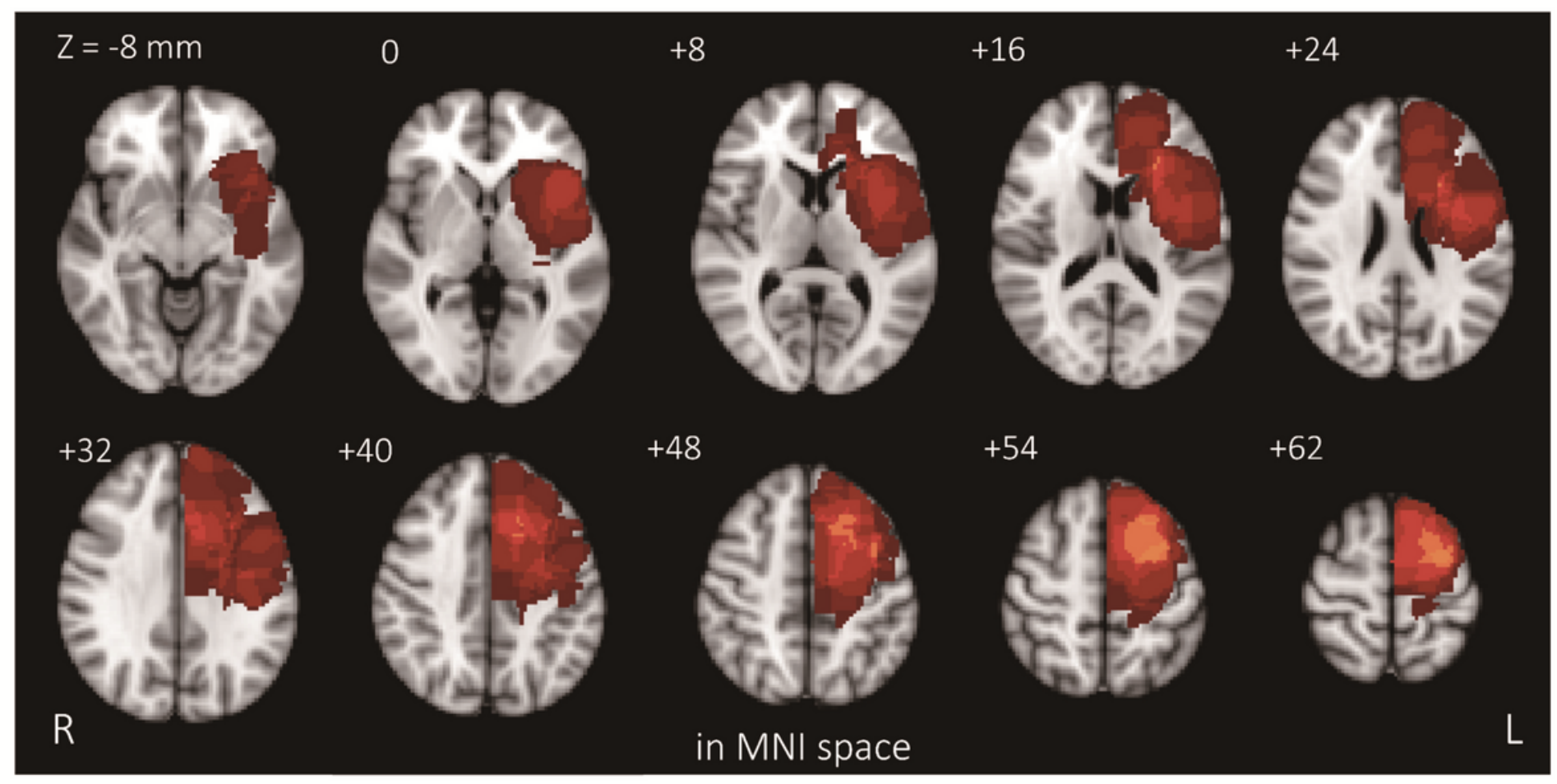

Number of Patients

\section{Figure 1}

Lesion topography of 18 patients with left frontal glioma in Montreal Neurological Institute (MNI) space.

Each voxel was identified as part of the tumor region from at least one patient. The color bar represents the number of patients with a lesion on a specific voxel. 


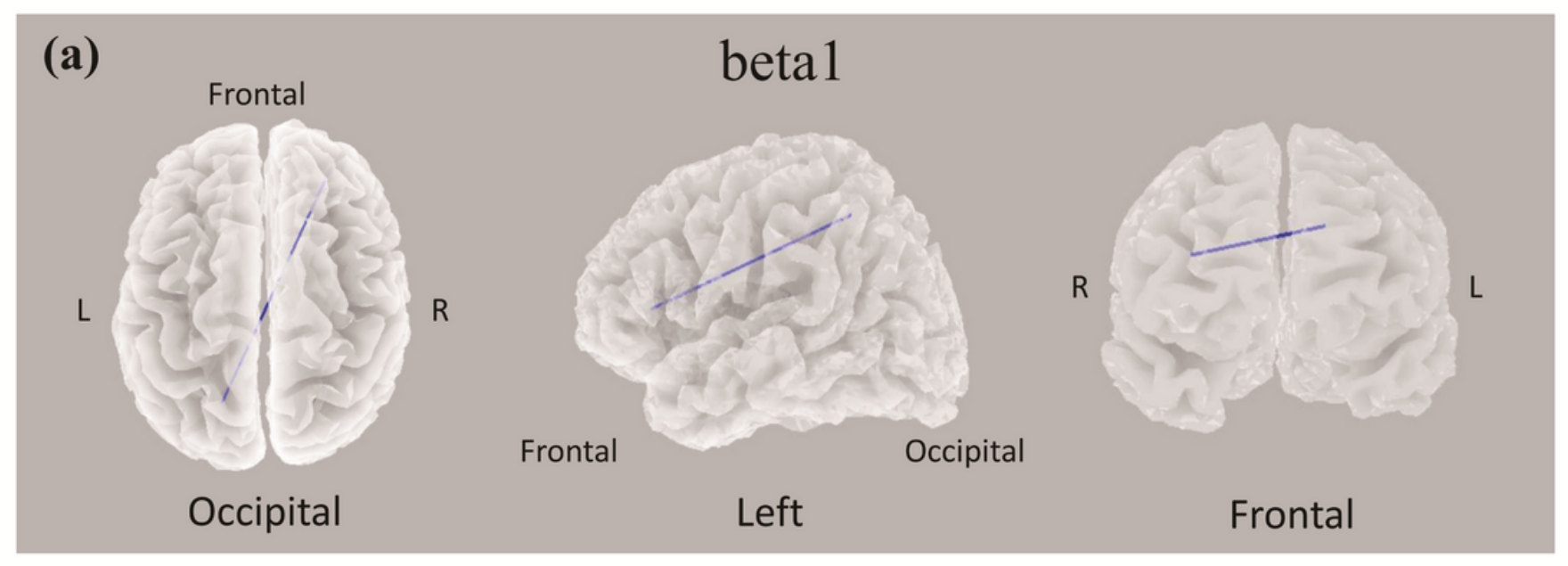

(b)
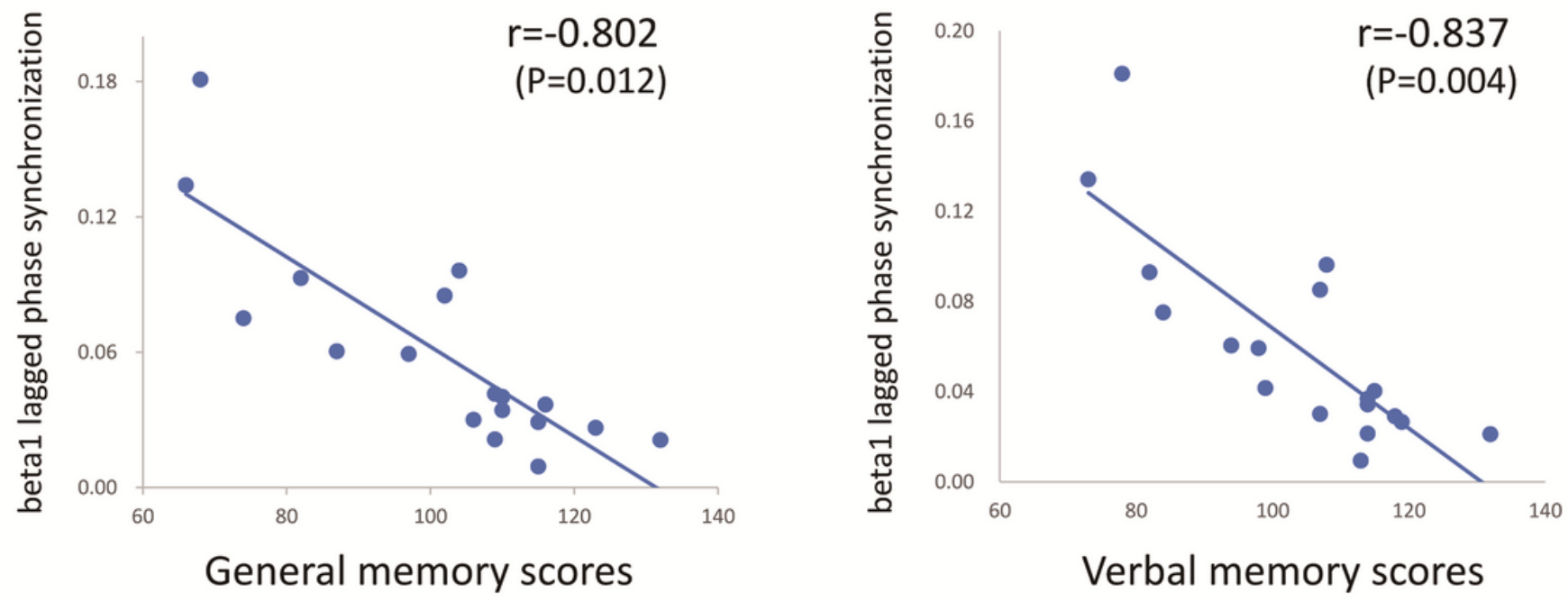

Figure 2

Association between lagged phase synchronization and general memory (GM) and verbal memory score by ELORETA.

a) eLORETA wire diagram of the right anterior prefrontal cortex (raPFC) and left superior parietal lobule (ISPL) show a significant negative correlation with GM and verbal memory scores in beta1 lagged phase synchronization (LPS). Blue line indicates connectivity between raPFC and ISPL, with a significant negative correlation between GM and veral memory scores and beta1 LPS. b) Scatter plot of GM and beta1 LPS values, verbal memory scores and beta1 LPS values intensities for 18 subjects. GM and verbal memory scores show negatively strong correlation with LPS value between raPFC and ISPL in the beta1 band (GM score $\mathrm{R}=-0.802, \mathrm{P}=0.012$; Verbal memory score $\mathrm{R}=-0.837, \mathrm{P}=0.004$ ). 